\title{
Mobile-Phone Based Chemical Analysis - Instrumental Innovations and Smartphone Apps
}

\section{Marek Trojanowicz*}

Department of Chemistry, Institute of Nuclear Chemistry and Technology, University of Warsaw, Warsaw, Poland

\begin{abstract}
Main trends in development of analytical instrumentation involve design a measuring instruments, which are able to provide the largest possible amount of analytical information about analyzed material, but simultaneously also simplification and scaling-down analytical instruments towards a possible use directly by users without specialized training. Both those development trends are associated with another important tendency, which is the elimination of direct human effort in carrying out different operations in chemical analytical procedures by design of measuring systems, which are mechanized, robotized, or even completely automated involving various contributions of artificial intelligence. One of elements used also for further instrumental progress, is in recent decade the application of mobile phones in further modification of analytical devices for remote and personal use. The potential utility value of a concept to equip mobile-phones in chemical sensors can be considered as a very efficient way for improving the access to a personal analytical devices for instance in bioanalytical applications, smartphone-based biosensors, and integration of microfluidic devices and smartphones.
\end{abstract}

Keywords: Chemical analysis; Smart phone apps; Analytical instrumentation; Innovations

\section{Introduction}

All along the development of analytical chemistry is a function of progress in all areas of chemistry, and also other disciplines of science and natural sciences, and progress in various technologies. A particularly essential element of progress in analytical chemistry and chemical analysis, developed for the needs of different branches of science and technology, is observed for a more than century evolution of measuring instrumentation. Since beginning of development of instrumental chemical analysis, up to enormous progress witnessed in recent decades in the evolution of instrumentation, one can observe two apparently contradictory tendencies. On the one hand, there is a trend to design a measuring instruments (setups), which are able to provide the largest possible amount of analytical information about analyzed material, but they can be operated by highly-trained personnel only and require specialized laboratories of advanced infrastructure. On the other hand, especially last decades, bring huge progress in simplification and scaling-down analytical instruments towards a possible use directly by users without specialized training, for instance by patient using simplified personal diagnostic tools, a farmer controlling acidity of soil or the level of a pesticide residue in soil, or miner equipped with personal dosimeter for the monitoring the level of toxic gases in the workplace. Both those development trends are associated with another important tendency resulting from a general technological progress in all fields of contemporary industry. It is the elimination of direct human effort in carrying out different operations in chemical analytical procedures by design of measuring systems, which are mechanized, robotized (see e.g., robotics-assisted mass spectrometry setup [1], or even completely automated involving various contributions of artificial intelligence [2]. It has a tremendous effect on efficiency and yield of conducted analytical measurements, and significantly supporting performing them under hazardous conditions. Undoubtedly, a very crucial driving force for the development of analytical instrumentation is constantly increasing demand on more and more detailed and complete information about use materials in practically all disciplines of modern science and technology, health care, environmental protection, food control and quality testing of different materials. Generally, the scaling-down of analytical instrumentation is observed since the middle of previous century, which takes place parallel to revolutionary progress in electronics, micromechanics, material and computer science, and additional impulse to this evolution was given in last two decades by landslide development of nanotechnology. Well recognized target driving this trend for long decades was a need of construction of analytical instrumentation for field applications with remote control oriented towards environmental and process control applications, for controlling operation of transportation devices, various industrial installations, not mentioning also analytical instrumentation for space missions, e.g., the wet chemistry laboratory with an array of sensors designed for the 2007 Phoenix Mars Scout Lander [3]. This is often associated with invention of a totally new concepts of design of analytical instruments, where the example can be, for instance thin-layer slide analyzers developed for clinical analysis [4]. In a scaled-down format there are produced and employed nowadays the most sophisticated and complex analytical instruments such as chromatographs, different types of absorption, fluorescence, resonance and mass spectrometers, and multicomponent chemical analyzers for clinical diagnostics. Simultaneously, last decades brought outstanding progress in design of miniaturized chemical sensors and biosensors, based on the application of different concepts of signal transduction, involving electrochemical, optical, thermal or masssensing relationships and phenomena. They provide fundaments for the development of portable analytical instruments for personal use, including numerous non-invasive sensing devices for personal care, sensors employed widely for environmental control and work place monitoring, even in a wearable format. All those approaches are broadly supported by modern miniaturized electronics and micromechanics. A unique civilization achievement in last decades is unprecedented progress in electrical and signal transmission and communication, including miniaturization of telecommunication devices (mobile phones, smartphones), and global access to countless data bases based

Corresponding author: Marek T, Department of Chemistry, Institute of Nuclear Chemistry and Technology, University of Warsaw, Warsaw, Poland, Tel: 48225041030; E-mail: trojan@chem.uw.edu.pl

Received May 26, 2017; Accepted June 06, 2017; Published June 12, 2017

Citation: Trojanowicz M (2017) Mobile-Phone Based Chemical Analysis Instrumental Innovations and Smartphone Apps. Mod Chem Appl 5: 220. doi: 10.4172/2329-6798.1000220

Copyright: ( 2017 Trojanowicz M. This is an open-access article distributed under the terms of the Creative Commons Attribution License, which permits unrestricted use, distribution, and reproduction in any medium, provided the original author and source are credited. 
on satellite signal transmission and common use of the Internet. One of the most commonly used element of that revolutionary progress mobile phones - are in recent decade increasingly utilized in further modification of analytical devices for remote and personal use. In fact mobile-phones are already equipped with numerous physical sensors such as microphones, light detectors, cameras, proximity sensors or modules for GPS. The potential utility value of a concept to equip them also in chemical sensors can be considered as a very efficient way for improving the access to a personal analytical devices, which can be convincingly illustrated by the fact, that already in 2015 about 7 billion of mobile phones was exploited globally, while forecasted number of mobile phone users in 2018 is almost 5 billion [5]. An incredibly fast development of that mobile sensing field, which is observed in less than one decade, was already subject of numerous review papers in scientific journals. Recent ones are devoted, for instance to bioanalytical applications [6], smartphone-based biosensors [7], and integration of microfluidic devices and smartphones [8].

A basic advantage of the application of mobile phones for analytical purposes is the possibility of using built-in cameras for detection and recording of different optical signals, and also electrical signals generated by integrated detecting/transducing devices and their further transmission using cellular telecommunication. The application of smartphones, as the latest generation of mobile phones with multicore processors and touch screen operations, and with the use of additional applications, enables also a real-time signal processing and a good quality display. This is a reason of such rapid grow of interest in such a way of instrumentation and simultaneously miniaturization of analytical procedures. Especially crucial achievement is also parallel miniaturization of mobile phone-integrated optical devices for portable imaging for the needs of point-of-care clinical diagnostics. The lightweight microscope can be mounted on the phone without any additional lenses. Besides that microscopic imaging, smartphone-based systems are also designed to detect some macroscopic changes in length and width e.g., for blood typing with the use of a barcode-like paper sensor [9]. This approach of hyphenating the paper-based sensor with mobile phone for the detection of analytical signal was preceded several years earlier by the design of paper-based microfluidic devices for simple telemedicine application dedicated for developing countries. In those setups camera phones were successfully employed for digitizing the color intensity in colorimetric assay, with further transmission of digital information [10]. Those abovementioned applications to clinical diagnostics and many other developed devices reported in the literature, confirm many facts from the history of analytical instrumentation in last 6-7 centuries, that very many breakthroughs in design of innovative analytical instruments is created in the field of clinical applications. This can be exemplified by instruments for laboratory flow analysis of various generation, discrete analyzers, centrifugal analyzers, mentioned already strips designed for various detection techniques or lateral flow strips. Similarly first developments of mobile phone-based analytical setups are predominantly addressed for clinical applications [6]. It has to admitted, however, that a certain complication for very broad application of such devices can be a sampling step, if it has to be a whole blood or plasma, although the common acceptance by users of e.g., invasive electrochemical or optical glucometers proves, that in practice it is possible. Obviously such a problem does not exist for other clinical samples such as urine, saliva or sweat. In fact one of the first commercialized mobile phone-based devices is a glucometer equipped with electrochemical sensor for analysis of whole blood. As another commercialized device can be mentioned the analyzer for detection of volatile sulfur compounds in breath, which are generated by bacteria biofilms in mouth. Two examples of such commercialized devices are showed in Figure 1. This outstanding, rapid progress in design of such systems results in increasingly sophisticated constructions, dedicated to different field of analytical applications, of which several will be presented briefly below. For medical purposes a microfluidic-based smartphone dongle with cassette with five optical detection zones was developed for simultaneous colorimetric determination of hemoglobin and detection of HIV antibodies with an immunoassay, based on silver precipitation on gold colloids [11] (Figure 2A and 2B). In instrumentally much simpler measuring system, the colorimetric detection employing silver nanoparticles was reported for the determination of local anesthetic lidocaine. It was based on the reaction carried out in Eppendorf vials, collecting the signal images in a safety cabinet by smartphone, and further signal processing in Adobe Photoshop software [12]. A much more sophisticated smartphone-based colorimetric system was recently reported and tested for the determination of human albumin [13]. In this case, a three-dimensional printed centrifugal microfluidics, mounted on a microcentrifuge was designed for ELISA immunosorbent assay with smartphone-based measurements. Also microfluidic chip, but with four arrays of 15 reaction wells was developed for carrying out a smartphone-based genetic testing, employing fluorescent DNA binding dye [14].
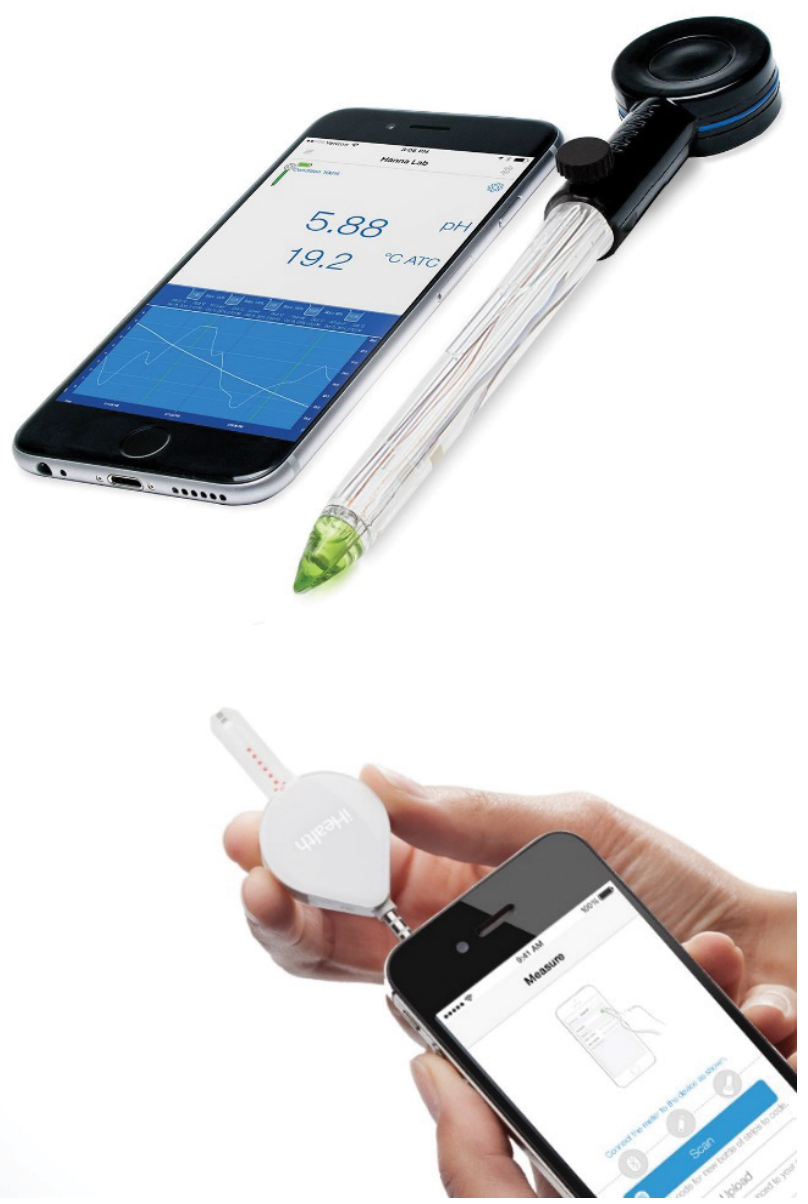

Figure 1: The examples of commercially available mobile-phone-based portable analytical devices: A - The $\mathrm{pH}$ probe with Bluetooth technology (http://hannainst.com/halo), B - The electrochemical portable glucose meter employing blood glucose test stripe, which plugs directly into the smartphone's headphone jack, and displays results directly on the phone screen [11]. 
Employing a loop-mediated isothermal amplification, a simultaneous analysis of four samples can be performed, each for multiple genetic markers in parallel. Its performance was tested using bacterial DNA and the developed system presents enormous potential for point-ofcare genetic testing. Among smartphone-based analytical systems for other than clinical applications, one can find, for instance, a developed in recent years the mobile phone attachment for the detection of specific markers in milk (Figure 2C). It detects antibodies, of which presence indicates a illegally used factor increasing milk production by cows [15]. The determination was carried out by fluorescence immunoassay employing quantum dots (QDs) coupled detection antibodies. The emitted fluorescence was detected using the mobile phone camera and processed using a custom-made Android application. The quenching of QDs fluorescence was employed for the determination of arsenic in surface waters, and the application of a mobile phone camera for such measurements was demonstrated [16]. Another example of environmental application of smartphone-based analytical approach was the determination of pesticide residues involving acetyl cholinesterase inhibition and colorimetric determination of a yellow product [17]. Instrumentally, the developed barcode assay, involved the use of polymeric chip with reaction channel situated under a printed barcode, which can be read by a scanning app installed on a smartphone. Although there are already available several commercial applications of such systems to mobile diagnostics, the majority of published works in this field are still rather proof-ofconcept reports. Further developments in this field are truly challenging. The majority of reported designs use the camera as a detector system for photometry, reflectance spectrometry or luminescence, but the drawback of mobile-phone cameras is that they do not provide direct pixel intensity values. In case of systems with electrochemical detections, some special devices integrating the mobile-phones with detection devices, transducers and electrodes are needed. A larger attention should be focused on design of measuring systems that do not require the use of tracers or reagents for the analyte detection. In systems hyphenating the mobile-phones with microfluidics the future developments must address the introduction of the sample, and application of on-chip valves and pumps for fluidic transport. As limitation of the use of those systems, generally, has to be admitted a poor signal transmission and signal quality still taking place in many low-resource areas, which may practically affect the use of such systems. Besides challenging instrumental innovations developed for application with mobile phones, a powerful modern smartphones can be nowadays employed in research and academic practice as a pocket computer with numerous useful applications, instead of laptops or desk computers. Mobile software applications ("apps") provide different functions and possibilities, and are especially convenient educational tools. Of great importance for progress in this field is the development of a cloud computing technology, which offers a wide access through cloud to numerous applications and enable storage of a large volume data and is very helpful e.g., in e-learning platforms. The mobile apps widely available in recent years can be grouped in reference/study guides, molecular viewers, research tools, utilities for laboratory experimentation and periodic table helpers [18]. Many of available apps can be very helpful in analytical chemistry study and research. In order just to mention few examples: ChemSpider providing access to chemical database of the Royal Society of Chemistry, UK, Chemistry Helper among different functions provides access to spectroscopy tables of IR and $\mathrm{H}_{1}$ and $\mathrm{C}_{13} \mathrm{NMR}$, LC Calculator helping in optimizing liquid chromatography separation, and Analytical Chemistry Challenges providing a unique way to assess the knowledge of basic and instrumental analytical chemistry. All those instrumental and software innovations are convincing evidences of continuous progress in chemical education and modernization of analytical instrumentation, which facilitates the use of chemical analysis in various areas of contemporary human activity.
A

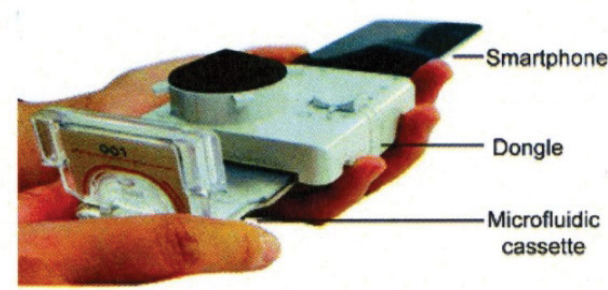

a

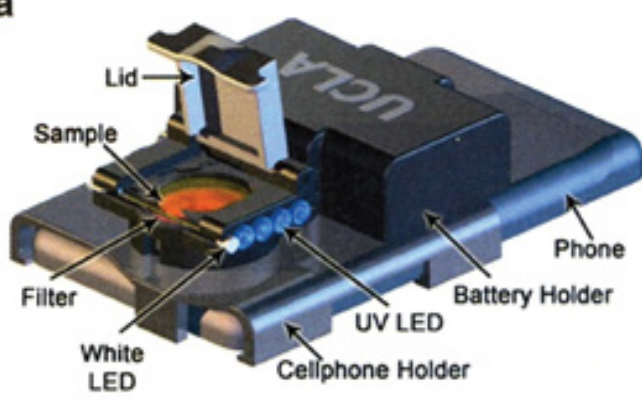

B

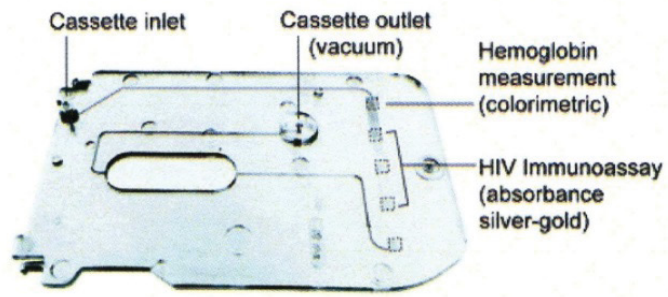

Figure 2: Two examples of mobile-phone-based systems attachments for optical detections: A - Photograph of smartphone dongle for haemoglobin and HIV antibodies detection, and B - photograph of employed microfluidic cassette with testing zones [13], C - Schematic overview of the mobile-phone attachment for fluorescent immunoassay of rbST biomarker in milk extracts [17]. 
Citation: Trojanowicz M (2017) Mobile-Phone Based Chemical Analysis - Instrumental Innovations and Smartphone Apps. Mod Chem Appl 5: 220. doi: $10.4172 / 2329-6798.1000220$

\section{References}

1. Chiu S, Urban PL (2015) Robotics-assisted mass spectrometry assay platform enable by open-source electronics platform. Biosensors Bioelectron 64: 260268.

2. Parbhu GRD, Urban PL (2017) The dawn of unmanned analytical laboratories. Trends Anal Chem 88: 41-52.

3. Kounaves SP, Hecht MH, West SJ, Morookian JM (2009) The MECA we chemistry laboratory on the 2007 Phoenix Mars Scout Lander. J Geophys Res 114: E00A19.

4. Curme H, Rand RN (1997) Early history of Eastman Kodak Ekta chem slides and instrumentation. Clin Chem 43: 1647-1652.

5. Number of Mobile Phone Users Worldwide from 2013 to 2019 (in Billions).

6. Vashist SK, Mudanyali O, Schneider EM (2014) Cellphone-based devices for bioanalytical sciences. Anal Bioanal Chem 406: 3263-3277.

7. Roda A, Michelini E, Zangheri M, Fusco M (2016) Smartphone-based biosensors: A critical review and perspectives. Trends Anal Chem 79: 317-325.

8. Yang K, Peretz SH, Liu Y, Lin F (2017) Novel developments in mobile sensing based on the integration of microfluidic devices and smartphones. Lab Chip 16: $943-958$.

9. Guan L, Tian J, Cao R, Li M (2014) Barcode-like paper sensor for smart-phone diagnostics: An application of blood typing. Anal Chem 86: 11362-11367.

10. Martinez AW, Phillips ST, Carrilho E, Thomas SW (2008) Simple telemedicine for developing regions: Camera phones and paper-based microfluidic devices for real-time off-site diagnosis. Anal Chem 80: 3699-3707.

11. Guo T, Patnaik T, Kuhlmann K (2015) Smartphone dongle for simultaneous measurement of hemoglobin concentration and detection of HIV antibodies. Lab Chip 115: 3514-3520.

12. Ansari N, Lodha A, Pandya A, Sutariya PG (2015) Lab-on-phone citrate-capped silver nanosensor for lidocaine hydrochloride detection from a biological matrix. Anal Meth 7: 9084-9091.

13. Ukita $Y$, Utsumi $Y$, Takamura $Y$ (2016) Direct digital manufacturing of mini-centrifuge-driven centrifugal microfluidic device and demonstration of a smartphone-based colorimetric enzyme-linked immunosorbent assay. Anal Meth 8: 256-262.

14. Stedfeld RD, Tourlousse DM, Seyrig G, Stedfeld TM (2012) Gene-Z: A device for point of care genetic testing using a smartphone. Lab Chip 12: 1454-1462.

15. Ludwig SKJ, Zhu H, Phillips S, Shiledar A (2014) Cellphone-based detection platform for rbST biomarker analysis in milk extracts using a microsphere fluorescence immunoassay. Anal Bio anal Chem 406: 6857-6866.

16. de Villiers CA, Lapsley MC, Hall EAH (2015) A step towards arsenic measurement for surface waters. Analyst 140: 2644-2655.

17. Guo J, Wong JXH, Cui C (2015) A smartphone-readable barcode assay for the detection and quantitation of pesticide residues. Analyst 140: 5518-5525.

18. Libman D, Huang $L$ (2013) Chemistry on the go: Review of chemistry apps on smartphones. J Chem Educ 90: 320-325. 